University of Nebraska - Lincoln

DigitalCommons@University of Nebraska - Lincoln

Faculty Publications, Department of Psychology

Psychology, Department of

January 1995

\title{
Morphine Taste Conditioning and Analgesia: Assessing Conditioned and Novelty-Induced Analgesia
}

Rick A. Bevins

University of Nebraska-Lincoln, rbevins1@unl.edu

Joanne M. Valone

University of Kentucky

Melinda C. Bradley

University of Kentucky

Michael T. Bardo

University of Kentucky

Follow this and additional works at: https://digitalcommons.unl.edu/psychfacpub

Part of the Psychiatry and Psychology Commons

Bevins, Rick A.; Valone, Joanne M.; Bradley, Melinda C.; and Bardo, Michael T., "Morphine Taste Conditioning and Analgesia: Assessing Conditioned and Novelty-Induced Analgesia" (1995). Faculty Publications, Department of Psychology. 290.

https://digitalcommons.unl.edu/psychfacpub/290

This Article is brought to you for free and open access by the Psychology, Department of at DigitalCommons@University of Nebraska - Lincoln. It has been accepted for inclusion in Faculty Publications, Department of Psychology by an authorized administrator of DigitalCommons@University of Nebraska - Lincoln. 
Published in Experimental and Clinical Psychopharmacology, 3:1 (1995), pp. 9-14; doi: 10.1037/1064-1297.3.1.9

Copyright (C) 1995 American Psychological Association. Used by permission. "This article may not exactly replicate the final version published in the APA journal. It is not the copy of record.” http://www.apa.org/journals/

\title{
Morphine Taste Conditioning and Analgesia: Assessing Conditioned and Novelty-Induced Analgesia
}

\author{
Rick A. Bevins, Joanne M. Valone, Melinda C. Bradley, Michael T. Bardo \\ Department of Psychology, University of Kentucky
}

\begin{abstract}
In previous work showing a taste-elicited decrease in pain sensitivity (J. S. Miller, K. S. Kelly, J. L. Neisewander, D. F. McCoy, \& M. T. Bardo, 1990), the rats (Rattus norvegicus) were always habituated to an inactive hot plate after each drug injection. The present report examined whether the analgesic response was a conditioned response to the taste or a response to the novelty of the hot plate resulting from morphine disrupting the habituation process. In 3 experiments, it was found that hot plate novelty was mainly responsible for the analgesic response. For example, increasing the number of conditioning trials did not enhance analgesia in morphine-treated rats. Rather, it attenuated analgesia in saline-treated controls (habituation). Also, rats given habituation in a drug-free state failed to show an analgesic response compared with controls.
\end{abstract}

Recent work pairing a taste conditioned stimulus (CS) with a morphine unconditioned stimulus (US) has reported evidence for an analgesic conditioned response (CR) to the taste (Bardo \& Valone, 1994; Miller, Kelly, Neisewander, McCoy, \& Bardo, 1990). In that work, rats were given 15-min access to a saccharin taste CS followed either by an injection of morphine $(15 \mathrm{mg} / \mathrm{kg})$ or saline. Thirty min after the injection, each rat was placed on the inactive hot plate for $60 \mathrm{~s}$ to habituate it to the hot plate environment and the testing procedures. These procedures were repeated for several conditioning trials. On the test day, all rats received the taste CS alone (no morphine) for $15 \mathrm{~min}$. Thirty min after CS exposure, each rat was assessed for pain responsivity on an active hot plate (e.g., $54{ }^{\circ} \mathrm{C}$ ). Rats given saccharin paired with morphine showed analgesia (longer paw-lick latencies) relative to saline-injected controls. Moreover, controls that received morphine explicitly unpaired with the taste did not show the analgesic response (Miller et al., 1990). This result suggests that the analgesic response is associative (i.e., a conditioned response). Subsequent work has shown that stress-induced analgesia produced by the morphine-conditioned taste aversion was not responsible for the analgesia because rats given taste aversion conditioning with lithium failed to display longer paw-lick latencies relative to controls (Bardo \& Valone, 1994).

The demonstration of an analgesic CR to a taste cue may have important implications for theories of drug abuse (e.g., O'Brien, Ehrman, \& Ternes, 1986) and conditioned drug effects (e.g., Stewart, 1992). For example, in some situations morphine may condition an analgesic response (Miller et al., 1990), whereas in other situations morphine may condition a hyperalgesic response (Krank, Hinson, \& Siegel, 1981; Siegel, 1975). Theories of drug conditioning

This work was supported by U.S. Public Health Service Grants DA05312 and DA07746.

Corresponding author: Rick A. Bevins will need to develop a priori methods for predicting the form of the conditioned response based on the CS modality and the drug US. Also, it will be necessary for theories to elucidate the role of these differing CR forms. The taste-elicited analgesic response may also have clinical relevance. Bardo and Valone (1994) suggested that the analgesic conditioned response conditioned to the taste cues may be useful for enhancing morphine-induced analgesia or for slowing the development of tolerance to morphine's analgesic effect in a clinical setting (e.g., postsurgical pain).

Given the potential theoretical and clinical significance of the taste-conditioning results, it is important that we determine whether the analgesic response is a conditioned decrease in pain sensitivity to the taste CS or an artifact of the conditioning procedures. The aim of the present report is to examine an alternative interpretation to the conditioned response account based on the phenomenon referred to as novelty-induced analgesia. Novelty-induced analgesia is defined as the tendency for rats to show a decrease in pain sensitivity in novel environments (Bardo \& Hughes, 1979; Foo \& Westbrook, 1991; Rochford \& Dawes, 1993; Sherman, 1979). In the taste-conditioning work, morphine may protect the novelty of the hot plate and test procedures by interfering with the habituation process on conditioning days (Bardo \& Hughes, 1979; Foo \& Westbrook, 1991; Rochford \& Dawes, 1993; Rochford \& Stewart, 1987). That is, to habituate the rats to the testing procedures, an experimenter exposes the rat to the inactive hot plate $30 \mathrm{~min}$ after injection. The morphine-injected rats are under the influence of morphine at the time of this habituation. Therefore, the test day represents the first time the morphine-treated rats experience the hot plate without morphine. If morphine is preventing habituation, the novelty of the hot plate and testing procedures could serve to increase paw-lick latencies (i.e., novelty-induced analgesia) relative to saline-treated controls that were able to habituate to the testing situation (Bardo \& Hughes, 1979; Espejo, Stinus, Cador, \& Mir, 1994). 


\section{Experiment 1}

The purpose of the present work was to assess the role of conditioned and novelty-induced analgesia in the taste-conditioning work described earlier. In Experiment 1, we did so by varying the number of trials (or number and total amount of exposure to the hot plate) before testing pain responsivity. In general, conditioned responding tends to strengthen with the number of conditioning trials (Mazur, 1994; Pavlov, 1927). Hence, according to the conditioned response account, we would expect an increase in paw-lick latencies with more conditioning trials (one, three, or six). The novelty account does not predict an increase in the analgesic response. If anything, the novelty account predicts a decrease in paw-lick latencies with greater exposure to the hot plate (1 min for each trial). This prediction, however, depends on the ability of morphine to attenuate habituation to novel stimuli in the hot plate and testing situation.

\section{Method}

Animals. The subjects were 60 male Sprague-Dawley rats (Rattus norvegicus), 200-225 g on arrival from Harlan Industries (Indianapolis, IN). They were housed individually in hanging wire-mesh cages in a colony room on a $12 \mathrm{~h}$ light-dark cycle. All experiments in the present report were conducted during the light phase of this cycle. Food was always available in the home cages. Water access was restricted as described below. Rats were handled on each of the 2 days preceding the start of the experiment.

Apparatus. Fluid presentations occurred in a rack of hanging wiremesh cages $(24.5 \times 17.5 \times 17.5 \mathrm{~cm}$ high $)$ similar to the home cages. Consumption was measured to the nearest milliliter using $100-\mathrm{ml}$ graduated drinking tubes attached to the front of each cage. A slide warming tray (Model 26020, Clinical Scientific Equipment Co., Melrose Park, IL) was used as a hot plate apparatus. A $20 \times 15 \times 28 \mathrm{~cm}$ high clear Plexiglas box with no floor or top was placed on the tray to prevent the rat from escaping the surface of the hot plate.

Drugs. Morphine sulfate (National Institute on Drug Abuse, Rockville, MD) was dissolved in saline $(0.9 \% \mathrm{NaCl})$. All injections were subcutaneous and the dosage was calculated based on the salt form of the drug.

Procedure. Rats were water deprived for 6 days before conditioning. On these days, they received 15-min access to water in the rack of cages with the drinking tubes. Following Day 6, rats were randomly assigned to treatment groups ( $n=9-11$ per group) with the restriction that water intake on Day 6 did not differ statistically among groups. On Day 7, rats were given the first conditioning trial. Conditioning consisted of $15-\mathrm{min}$ access to a $0.1 \%(\mathrm{w} / \mathrm{v})$ sodium saccharin solution followed by an injection of morphine $(15 \mathrm{mg} / \mathrm{kg})$ or saline. Thirty min after the injection, rats were placed on the inactive hot plate $\left(22^{\circ} \mathrm{C}\right)$ for $60 \mathrm{~s}$. On the next 2 days (Days 8 and 9), rats received 15-min access to water without an injection. For the groups that received three or six conditioning trials, Days 7, 8, and 9 were repeated for the appropriate number of trials. Thus, the design of Experiment 1 was a $3 \times 2$ between-subjects factorial design in which the number of trials before analgesia testing (one, three, or six) was crossed with drug (saline vs. morphine).

The hot plate test for pain responsivity was administered on the day after the last water access day. On the test day, each rat was given 15-min access to the saccharin CS followed immediately by an injection of saline (CS alone). The rat was then placed on the active hot plate $\left(54^{\circ} \mathrm{C}\right) 30 \mathrm{~min}$ after exposure to the taste CS. The la- tency to the first paw lick (front or hind) and the latency to the hind paw lick was recorded. Each rat was removed from the hot plate immediately after the hind paw lick occurred. All rats in the present experiment and throughout this report were removed from the hot plate within $35 \mathrm{~s}$ of being placed on it.

Data analysis. We first analyzed each dependent measure (saccharin intake, first paw-lick latency, and hind paw-lick latency) using between-groups analyses of variance (ANOVAs). A Dunnett's test was used for planned and post hoc comparisons between morphinetreated groups and saline-treated controls with equal sample sizes. A Dunn's test was used for comparisons with unequal sample sizes. For all statistical tests, we used a two-tailed rejection region of .05 .

\section{Results and Discussion}

Figure 1 A shows the mean saccharin intake for each treatment group. Relative to saline-treated controls (empty bars), rats that received saccharin paired with morphine (striped bars) consumed less fluid. This decrease in consumption appeared greater after three and six trials than after one trial. These impressions were confirmed by a significant main effect of drug (morphine vs. saline), $F(1,54)=59.62$, and number of trials (one, three, or six, $F(2,54)=5.04$. The Drug $\times$ Trial interaction was not significant, $F(2,54)=2.18$. Planned contrasts revealed that each morphinetreated group drank less saccharin than its comparable saline-injected control group.

Figure $1 \mathrm{~B}$ shows the mean latency to the first paw lick for each treatment group. The first paw-lick latencies for the morphine-treated groups were similar regardless of the number of trials before testing. However, progressively shorter latencies for the saline-treated controls occurred with an increase in the number of trials. Overall analysis revealed a significant main effect of drug, $F(1,54)=4.58$, but the main effect of trials, $F(2,54)=$ 1.7 , and the Drug $\times$ Trial interaction, $F(2,54)=2.63$, were not significant. The planned comparisons between each morphinetreated group and its respective saline-injected control found a significant difference only after six trials. To assess the apparent decrease in paw-lick latencies for the saline-treated groups, we performed a post hoc comparison between the groups tested after one and six trials. Saline-treated rats tested after six trials had significantly shorter first paw-lick latencies than rats tested after one trial, $F(1,18)=7.8$. Unlike first paw-lick latencies, analysis of hind paw-lick latencies did not reveal any differences among groups ( $F \mathrm{~s}<1.7$; data not shown).

According to the novelty-induced analgesia hypothesis, the diminishing analgesic response in the saline-treated groups reflects a decrease in the novelty of the hot plate and testing situation with greater exposure. Moreover, morphine appeared to protect the novelty of the hot plate in the morphine-treated groups because paw-lick latencies did not decline with more exposure. An interpretation based solely on conditioning to the taste CS does not anticipate the data pattern found here. A conditioning account would predict an increase in paw-lick latencies with an increase in the number of taste-morphine pairings.

\section{Experiment 2}

Like Experiment 1, Experiment 2 also manipulated the amount of exposure to the hot plate. On each conditioning day, rats were exposed either to the hot plate or to a different apparatus. One set 
of rats received three trials before testing (three 1-min hot plate exposures vs. none) and a second set received six trials (six 1min hot plate exposures vs. none). An account based solely on conditioned pain suppression to the taste CS predicts that the exposure manipulation should not affect the demonstration of an analgesic CR. However, if the novelty-induced analgesia hypothesis is correct, then we should find evidence for analgesia only in the hot plate exposure condition. Note that the novelty of the hot plate is equated for the saline- and morphine-treated groups exposed to the other apparatus. Thus, paw-lick latencies should be similar in these two groups.

\section{Method}

Animals. The subjects were 78 male Sprague-Dawley rats (40 in the three-trial condition and 38 in the six-trial condition) housed and maintained as described in Experiment 1.
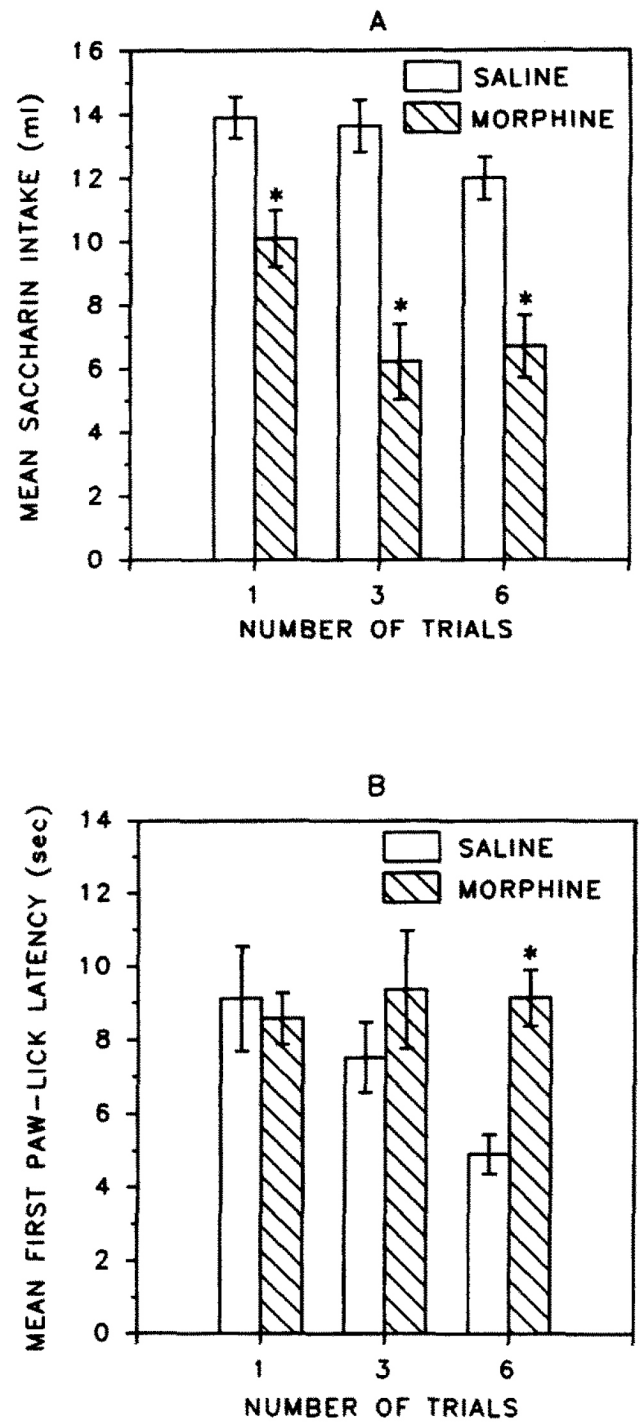

Figure 1. Mean consumption of saccharin conditioned stimulus (A) and mean first paw-lick latency (B) for each treatment group in Experiment 1. Separate groups were tested following one, three, or six taste-drug trials. Bars denote \pm 1 standard error of the mean and asterisks $(*)$ represent a statistically significant difference from the comparable saline control.
Apparatus. In addition to the hot plate and rack of cages that remained unchanged, a clear plastic maternity tub (Allentown Caging, Allentown, NJ) with the inside dimensions of $44 \times 24 \times 20.5 \mathrm{~cm}$ was used. The maternity tub was lined with pine wood chips, and a wire cover was used to prevent the rats from escaping.

Procedure. The conditioning and testing procedures were similar to Experiment 1 except that one group of rats was never exposed to the hot plate before the test day. Instead, they were placed for $60 \mathrm{~s}$ in the plastic maternity tub. By placing each rat in the maternity tub, we controlled for variables such as amount of handling and time out of home cage while varying whether rats had the opportunity to familiarize themselves with the hot plate. Thus, the design of Experiment 2 was a $2 \times 2$ factorial in which drug (saline vs. morphine) was crossed with exposure (hot plate [HP] vs. maternity cage $[\mathrm{MC}])$. Because the rats in the three-trial condition $(n=9-11$ per group) were received in a different shipment and were run separately from the rats in the six-trial condition ( $n=9-10$ per group), number of trials before testing was not treated as a factor in the data analyses.

\section{Results and Discussion}

Morphine-treated rats consumed less saccharin on the test day $(\mathrm{HP}=4.5 \mathrm{ml} ; \mathrm{MC}=6.0 \mathrm{ml})$ than saline-treated rats $(\mathrm{HP}=$ $13.4 \mathrm{ml} ; \mathrm{MC}=12.6 \mathrm{ml}$ ) following three trials. The same was true in the six-trial condition (for morphine-treated rats, HP = $6.2 \mathrm{ml}, \mathrm{MC}=5.2 \mathrm{ml}$; for saline-treated rats, $\mathrm{HP}=15.7 \mathrm{ml}, \mathrm{MC}$ $=15.3 \mathrm{ml}$ ). Whether rats were exposed to the hot plate or maternity cage had no effect on taste aversion. There was a significant main effect of drug in the three-trial condition, $F(1,36)=$ 102.45 , and the six-trial condition, $F(1,34)=71.15$. There was no main effect of exposure type (hot plate vs. maternity cage) and no Drug $\times$ Exposure Type interaction at three or six trials $(F \mathrm{~s}<2.36)$.

The left half of Figure 2 shows the mean first paw-lick latency in the three-trial condition. Following three trials, the main effect of drug, $F(1,36)=3.58, p=.067$, and exposure type, $F(1,36)=2.25, p=.095$, approached statistical significance. The Drug $\times$ Exposure Type interaction was not significant $(F<1)$. In the six-trial condition, these differences were enhanced (see right half of Figure 2). There was a significant main effect of drug, $F(1,34)=5.53$, denoting longer paw-lick latencies in the morphine-treated rats. Also, there was a significant effect of exposure type, $F(1,34)=6.99$, indicating overall longer first paw-lick latencies in the rats exposed to the maternity cage on each trial. The Drug $\times$ Exposure Type interaction was not significant $(F<1)$. Pair-wise comparisons revealed significantly longer first paw-lick latencies only in the morphinetreated rats exposed to the hot plate.

Analysis of hind paw-lick latencies revealed no statistical differences in the three-trial condition $(F \mathrm{~s}<1)$. In the six-trial condition, however, there was a main effect of exposure type, $F(1$, $33)=6.65$, denoting overall shorter hind paw-lick latencies by rats that received exposure to the hot plate $(M=8.17 \mathrm{~s}, S E M=$ $.62)$ than rats that were placed in the maternity cage $(M=10.86$ $\mathrm{s}, S E M=.85){ }^{1}$

\footnotetext{
${ }^{1}$ There are 33, instead of 34, degrees of freedom for the error term because one rat escaped from the hot plate before making a hind paw lick.
} 


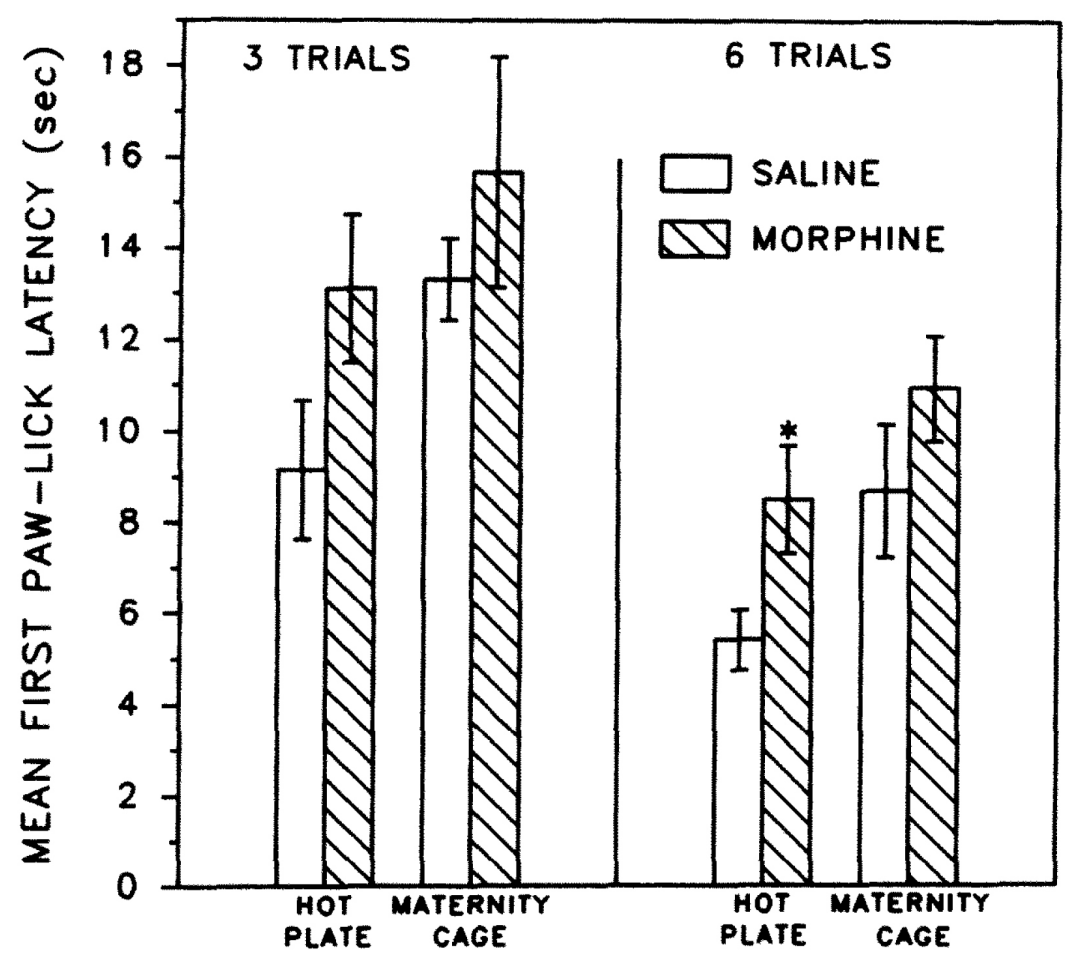

Figure 2. Mean first paw-lick latency following exposure to the inactive hot plate versus maternity cage for three trials (left half) or for six trials (right half) before testing in Experiment 2. Bars denote \pm 1 standard error of the mean and the asterisk $\left(^{*}\right)$ denotes a statistically significant difference from the comparable saline control.

Interestingly, this experiment found evidence for both a conditioned and a novelty-induced analgesic response. The lack of a Drug $\times$ Exposure interaction and the significant main effect of drug (i.e., overall longer paw-lick latencies in the morphinetreated rats) suggests that some conditioning did occur with six trials regardless of exposure type. This conclusion, however, was complicated by the a priori contrasts. Morphine-treated rats in the maternity cage condition had paw-lick latencies statistically comparable to the control group and to the morphine-treated rats exposed to the hot plate. This data pattern, and the lower overall paw-lick latencies in the hot plate condition, suggests a role of novelty-induced analgesia.

\section{Experiment 3}

Experiment 1 provided evidence for a novelty-induced analgesic response, whereas Experiment 2 suggested a possible role for both novelty-induced and conditioned analgesia. To further evaluate these two accounts, in Experiment 3 we equated total time on the hot plate (six 1-min exposures) while varying the day of exposure to the hot plate and testing situation. Rats were exposed either on the taste-drug conditioning day (usual procedure) or on an intervening water day without drug. The conditioning account predicts similar expression of an analgesic CR regardless of the day of hot plate exposure. On the other hand, the novelty interpretation predicts analgesia only in the morphine-treated rats exposed to the hot plate on the conditioning day.

\section{Method}

Animals and apparatus. The rats $(n=44)$ and apparatus were similar to those of Experiment 1.

Procedure. Conditioning and testing were similar to Experiment 1 except that we varied which day rats were habituated to the hot plate and testing procedures. One set of rats received $1 \mathrm{~min}$ of exposure to the hot plate on each conditioning day as described previously. Another set received exposure on the day before each conditioning trial (i.e., an intervening water day without drug). Hence, the design was a $2 \times 2$ factorial in which drug was crossed with day of hot plate exposure (conditioning vs. watering). There were six trials before testing ( $n=10-12$ rats per group).

\section{Results and Discussion}

Morphine-treated rats consumed significantly less saccharin than their respective controls, $F(1,40)=91.45$, regardless of whether placement on the hot plate occurred on the conditioning days $(6.1 \mathrm{vs} .15 .4 \mathrm{ml})$ or on the watering days $(6.7 \mathrm{vs} .16 .7 \mathrm{ml})$. The main effect of exposure day and the Drug $\times$ Day interaction was not significant $(F \mathrm{~s}<1)$. 
Figure 3 shows the mean first paw-lick latency data for rats that were exposed to the hot plate on each of the six conditioning days or on six watering days. Analyses revealed a significant main effect of drug, $F(1,40)=7.48$, and a significant Drug $\times$ Day (conditioning vs. watering) interaction, $F(1,40)=11.14$. The main effect of exposure day approached statistical significance, $F(1,40)=3.47 ; p=.07$. Planned comparisons found that only the morphine-treated group exposed to the hot plate on the conditioning day had longer first paw-lick latencies than their respective saline-injected control. Groups did not differ significantly in terms of their hind paw-lick latencies $(F \mathrm{~s}<1.9$; data not shown).

The lack of an analgesic response in morphine-treated rats exposed to the hot plate on watering days (no drug) was not anticipated by the conditioning account. This data pattern, however, does support a novelty-induced analgesia account. By exposing morphine-treated rats to the hot plate on a nonconditioning day, they, like saline-treated controls, had the same opportunity to become familiar with the hot plate and testing procedures. The similarity in paw-lick latencies between these two groups reflects the similarity in habituation to the hot plate at the time of testing.

\section{General Discussion}

Previous work has reported that a taste cue paired with morphine can elicit a conditioned analgesic response (Bardo \& Valone, 1994; Miller et al., 1990). The present experiments examined whether this decrease in pain sensitivity was a conditioned response to the taste $\mathrm{CS}$ or an analgesic response to the novelty of the hot plate resulting from morphine interfering with the habituation process (Bardo \& Hughes, 1979). Taken together, the results of the present report support the conclusion that the longer

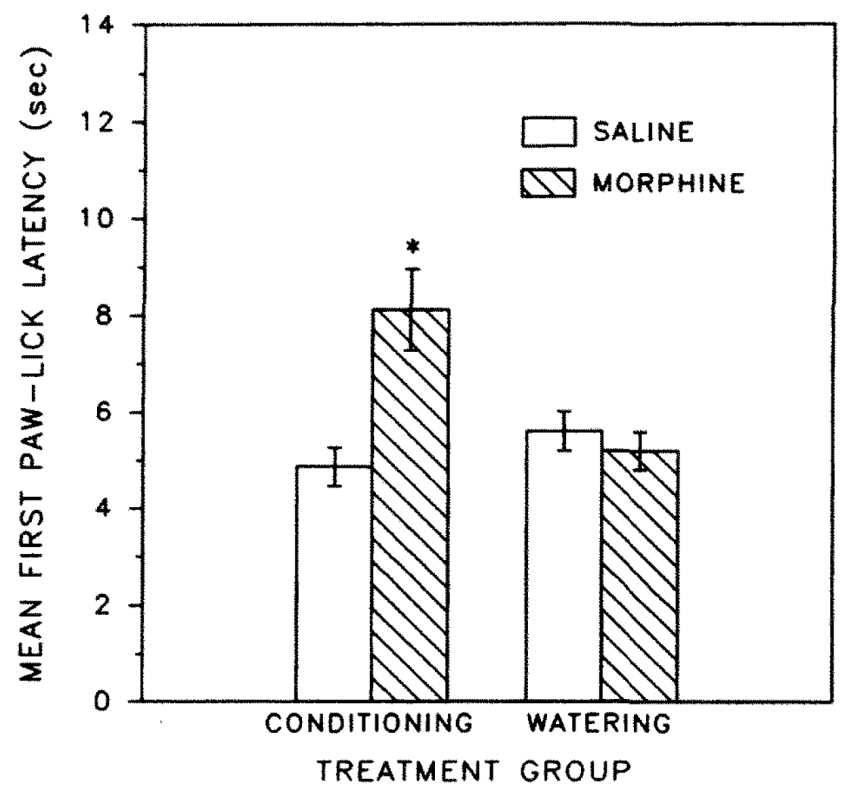

Figure 3. Mean first paw-lick latency following exposure to the inactive hot plate on six conditioning days versus six watering days in Experiment 3. Bars denote \pm 1 standard error of the mean and the asterisk (*) denotes a statistically significant difference from the comparable saline control. paw-lick latencies seen in morphine-treated rats are mainly due to novelty-induced analgesia rather than a conditioned analgesic response. Indeed, Experiments 1 and 3 found clear support for hot plate novelty as the source of the decrease in pain sensitivity.

The results of Experiment 2, however, appear to preclude us from concluding that novelty is the sole source of analgesia. That study found evidence for both a conditioned and a noveltyinduced analgesic response. The evidence for an analgesic CR in Experiment 2 may instead be the result of morphine interfering with the habituation process. That is, half the rats in that study were exposed to the plastic maternity tub $30 \mathrm{~min}$ after CS presentation. This maternity tub exposure, like hot plate exposure, may have served to habituate the rats to at least part of the testing procedures. For example, the saline-treated rats in the maternity tub condition were familiarized with being removed from the home cage, transported to the environment, and exposed to a novel situation. At the time of testing, the actual hot plate environment would be the only aspect of the testing procedure that was novel for the saline-treated rats. If morphine blocked the habituation process, then all aspects of the testing procedure would be novel for the morphine-treated rats. Because of the greater proportion of novelty for the morphine-treated rats, we would expect some evidence for analgesia (i.e., longer paw-lick latencies) in those rats.

Although the novelty-induced analgesia account appears to provide the most parsimonious explanation of the results observed here, one alternative account based on conditioning processes does exist. This alternative account requires two main assumptions. The first assumption is that when morphine-injected rats are exposed to the hot plate, that exposure serves to condition an analgesic response to the hot plate. Thus, the hot plate is a CS and the CR elicited by the hot-plate CS is a decrease in pain sensitivity. The second assumption is that the novelty of the hot plate initially produces analgesia but morphine does not protect it from habituation. Rather, morphine- and saline-treated rats exposed to the hot plate equally habituate to its novelty. This account would explain the results of Experiment 1 as follows: Habituation to the hot plate environment would account for the decrease in paw-lick latencies for the saline-injected controls. The long paw-lick latencies in the morphine-treated rats after one trial reflects novelty-induced analgesia. However, with repeated exposures to the hot plate, its novelty decreases. The paw-lick latencies do not decrease in this group because of the acquisition of an analgesic CR to the hot plate.

As for the results of Experiment 3, this alternative conditioning account would argue that morphine-treated rats exposed to the hot plate on watering days never received the hot plate paired with morphine. Thus, the conditioned analgesic response was never acquired to the hot plate CS. Moreover, given that these rats were exposed to the hot plate as much as the salinetreated controls, the novelty-induced analgesia was similarly decreased.

If this hot plate conditioning account is correct, then it poses at least one question. Why do we find conditioned analgesia to a context CS (i.e., hot plate) but others report hyperalgesia (Krank, 1987; Krank et al., 1981; Siegel, 1975) or no analgesia (Tiffany, Petrie, Baker, \& Dahl, 1983) to a context CS? Pro- 
viding an answer to this question is hindered by two problems. First, between-experiments comparisons, especially when conducted in different laboratories, are fraught with difficulties. Second, whereas the previous studies were designed to assess morphine tolerance, the present experiments did not address this issue. Therefore, it is not possible to directly compare among these studies.

The present work does not eliminate the possibility of a taste cue serving as a CS in a morphine-conditioned analgesia paradigm. Rather, the present series of experiments argues that the analgesic response seen in the present situation is better explained in terms of novelty-induced analgesia than in terms of conditioned responding to the taste CS. It is possible, however, that taste, which is readily associated with the aversive properties of a morphine US (see Figure $1 \mathrm{~A}$ ), may not be readily associated with the analgesic properties of morphine. Perhaps other stimuli such as odor or tactile cues would more readily serve as a CS in this situation. Clearly, further work exploring parameters such as CS duration or CS-US overlap (e.g., see Schwarz-Stevens \& Cunningham, 1993) is required to determine whether or not taste (or other cue modalities) can serve as a CS in a morphine-conditioned analgesia paradigm.

\section{References}

Bardo, M. T., \& Hughes, R. A. (1979). Exposure to a nonfunctional hot plate as a factor in the assessment of morphine-induced analgesia and analgesic tolerance in rats. Pharmacology Biochemistry and Behavior, 10, 481-485.

Bardo, M. T., \& Valone, J. M. (1994). Morphine-conditioned analgesia using a taste cue: Dissociation of taste aversion and analgesia. Psychopharmacology, 114, 269-274.

Espejo, E. F., Stinus, L., Cador, M., \& Mir, D. (1994). Effects of morphine and naloxone on behaviour in the hot plate test: An ethopharmacological study in the rat. Psychopharmacology, $113,500-510$.

Foo, H., \& Westbrook, R. F. (1991). Naloxone-induced hypoalgesia: Effects of heat, cold, and novelty. Quarterly Journal of Experimental Psychology, 43, 137-156.
Krank, M. D. (1987). Conditioned hyperalgesia depends on the pain sensitivity measure. Behavioral Neuroscience, 101, 854-857.

Krank, M. D., Hinson, R. E., \& Siegel, S. (1981). Conditioned hyperalgesia is elicited by environmental signals of morphine. Behavioral and Neural Biology, 32, 148-157.

Mazur, J. E. (1994). Learning and behavior (3rd ed.). Englewood Cliffs, NJ: Prentice Hall.

Miller, J. S., Kelly, K. S., Neisewander, J. L., McCoy, D. F., \& Bardo, M. T. (1990). Conditioning of morphine-induced taste aversion and analgesia. Psychopharmacology, 101, 472-480.

O’Brien, C. P., Ehrman, R. N., \& Ternes, J. W. (1986). Classical conditioning in human opioid dependence. In S. R. Goldberg \& I. P. Stolerman (Eds.), Behavioral analysis of drug dependence (pp. 329-356). New York: Academic Press.

Pavlov, I. P. (1927). Conditioned reflexes. New York: Dover.

Rochford, J., \& Dawes, P. (1993). Effect of naloxone on the habituation of novelty-induced hypoalgesia: The collateral inhibition hypothesis revisited. Pharmacology Biochemistry and Behavior, 46, 117-123.

Rochford, J., \& Stewart, J. (1987). Activation and expression of endogenous pain control mechanisms in rats given repeated nociceptive tests under the influence of naloxone. Behavioral Neuroscience, 101, 87-103.

Schwarz-Stevens, K. S., \& Cunningham, C. L. (1993). Pavlovian conditioning of heart rate and body temperature with morphine: Effects of CS duration. Behavioral Neuroscience, 107, 1039-1048.

Sherman, J. E. (1979). The effects of conditioning and novelty on rats' analgesic and pyretic response to morphine. Learning and Motivation, 10, 381-418.

Siegel, S. (1975). Evidence from rats that morphine tolerance is a learned response. Journal of Comparative and Physiological Psychology, 89, 498-506.

Stewart, J. (1992). Neurobiology of conditioning to drugs of abuse. In P. W. Kalivas \& H. H. Samson (Eds.), The neurobiology of drug and alcohol addiction (pp. 335-346). New York: The New York Academy of Sciences.

Tiffany, S. T., Petrie, E. C., Baker, T. B., \& Dahl, J. L. (1983). Conditioned morphine tolerance in the rat: Absence of a compensatory response and cross-tolerance with stress. Behavioral Neuroscience, 97, 335-353. 